\title{
Network Modeling of the U.S. Petrochemical Industry Under Raw Material and Hurricane Harvey Disruptions
}

\author{
Supporting Information
}

Sean E. DeRosa ${ }^{\not *}$ Yosuke Kimura, ${ }^{\ddagger \S}$ Mark A. Stadtherr,,${ }^{\phi}$ Gary McGaughey, ${ }^{\S}$ Elena McDonaldBuller, ${ }^{\S}$ David T. Allen ${ }^{\S}$

${ }^{\dagger}$ Sandia National Laboratories

${ }^{\S}$ Center for Energy and Environmental Resources, The University of Texas at Austin $\Phi$ McKetta Department of Chemical Engineering, The University of Texas at Austin

$\$$ These authors contributed equally.

*Author to whom correspondence should be addressed (E-mail: sean.derosa@utexas.edu)

\section{Contents}

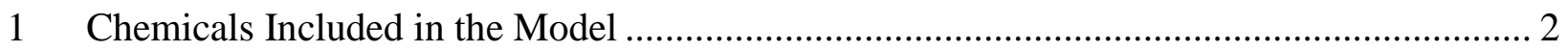

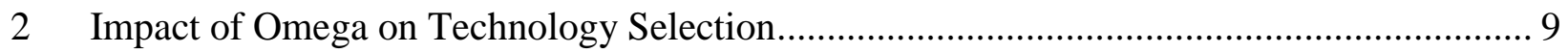

3 Modeled Effects of Assumed Capacity Disruptions by Geographic Storm Track: Expanded Results................................................................................................................. 10 


\section{Chemicals Included in the Model}

Table S1 shows the primary raw materials, Table S2 lists the unconstrained raw materials, and Table S3 lists all intermediate and final end products and their import/export status.

Table S1. Primary raw materials included in the model.

\begin{tabular}{|l|}
\hline crude oil \\
\hline ethane \\
\hline methane \\
\hline n-butane \\
\hline n-hexane \\
\hline n-pentane \\
\hline propane \\
\hline
\end{tabular}

Table S2. Unconstrained raw materials included in the model.

\begin{tabular}{|l|}
\hline AA-EGDA copolymer \\
\hline acetyl acetone \\
\hline activated carbon \\
\hline alumina \\
\hline alumina trihydrate \\
\hline aluminum chloride \\
\hline aluminum triethyl \\
\hline amberlite@ IRA-96 \\
\hline ammonium heptamolybdate \\
\hline ammonium molybdenum \\
\hline ammonium vanadate \\
\hline antimony trioxide \\
\hline antioxidant \\
\hline antioxidant, SBS \\
\hline ash disposal \\
\hline azobisisobutyronitrile \\
\hline benzoylperoxide \\
\hline boiler feed water \\
\hline butylated hydroxytoluene \\
\hline calcium carbide \\
\hline calcium chloride \\
\hline calcium hydroxide \\
\hline carbon monoxide \\
\hline carbon tetrachloride \\
\hline cation exchange resin \\
\hline
\end{tabular}




\begin{tabular}{|l|}
\hline caustic soda beads \\
\hline cetyl alcohol \\
\hline chain transfer agent \\
\hline chloroprene \\
\hline coagulant \\
\hline coal \\
\hline cobalt acetate-4H2O \\
\hline cobalt naphthenate \\
\hline corn \\
\hline cupric nitrate \\
\hline cuprous oxide \\
\hline 1,4-cyclohexanedimethanol \\
\hline di(2-ethylhexyl) \\
peroxidicarbonate (DEHPC) \\
\hline deionized water \\
\hline dibutyl fumarate \\
\hline dibutyl phthalate \\
\hline diisobutyl aluminium hydride \\
\hline dimethylamino benzaldehyde \\
\hline dimethyl formamide \\
\hline dinitrotoluene \\
\hline diphenyl ether \\
\hline enzymes \\
\hline ethanol \\
\hline $\begin{array}{l}\text { ethylene diamine tetra acetic } \\
\text { acid }\end{array}$ \\
\hline ethylidene norbornene \\
\hline fluorided silica-alumina \\
\hline formaldehyde \\
\hline formic acid \\
\hline GafacRE-610 \\
\hline glycerin \\
\hline hafnium acetate-4H2O \\
\hline hexamethyleneimine \\
\hline hydrazine, $85 \%$ \\
\hline hydrobromic acid, $48 \%$ \\
\hline hydrogen \\
\hline hydrogen chloride \\
\hline hydrogen cyanide \\
\hline hydroquinone \\
\hline inhibitors \\
\hline ion exchange resin \\
\hline
\end{tabular}




\begin{tabular}{|l|}
\hline iso-octyl thioglycolate \\
\hline isoprene \\
\hline ligand \\
\hline lime \\
\hline magnesium oxide \\
\hline manganese acetate-4H2O \\
\hline methylene chloride \\
\hline methylhydroquinone ether \\
\hline methylisobutyl ketone \\
\hline methylpropyl ketone \\
\hline MgO-KOH \\
\hline miscellaneous chemicals-SBR \\
\hline molybdenum powder \\
\hline n-butyllithium \\
\hline n-dodecyl mercaptan \\
\hline n-methyl-2-pyrrolidone \\
\hline n-octane \\
\hline n-propyl acetate \\
\hline naphthenic acid \\
\hline NCA activator \\
\hline nickel, raney \\
\hline nitric acid, 60\% \\
\hline nitrogen \\
\hline nylon waste \\
\hline o-dichlorobenzene \\
\hline oleum, 7\% \\
\hline oxalic acid \\
\hline oxygen \\
\hline p-nonylphenol \\
\hline p-t-butylphenol \\
\hline p-toluene sulfonic acid \\
\hline peroxide initiators \\
\hline phosgene \\
\hline phosphoric acid \\
\hline poly(vinylpyrrolidone) \\
\hline polymerization inhibitor \\
\hline potassium carbonate \\
\hline potassium hydroxide \\
\hline potassium oleate \\
\hline potassium persulfate \\
\hline rock salt \\
\hline
\end{tabular}




\begin{tabular}{|l|}
\hline scrubbing oil \\
\hline silica gel \\
\hline soap \\
\hline sodium acetate \\
\hline sodium carbonate \\
\hline sodium cetylsulfate \\
\hline sodium chloride \\
\hline sodium dihydrophosphate \\
\hline sodium pyrophosphate \\
\hline sodium sulfide, flake \\
\hline solvent \\
\hline sorbitanmonolaurate \\
\hline soybeanoil_epoxidized \\
\hline stabilizer, SBR \\
\hline sulfur dioxide \\
\hline sulfuric acid \\
\hline synthesis gas (2:1) \\
\hline t-butyl catechol \\
\hline t-butyl perbenzoate \\
\hline t-dodecylmercaptan \\
\hline TBPD chain transfer agent \\
\hline teal \\
\hline tri-n-butylamine \\
\hline triethylamine \\
\hline triethyl phosphate \\
\hline triisobutylaluminum \\
\hline trimethylacetic acid \\
\hline trimethylaluminum \\
\hline triphenylmethane \\
\hline tris(nonylphenol) phosphite \\
\hline vanadium trichloride \\
\hline
\end{tabular}

Table S3. Intermediate and final end products included in the model. If a material also has exports or imports it is noted in the second two columns.

\begin{tabular}{|l|c|c|}
\hline Chemical Name & Export & Import \\
\hline ABS resin & $\mathrm{X}$ & $\mathrm{X}$ \\
\hline acetaldehyde & $\mathrm{x}$ & \\
\hline acetic acid & $\mathrm{x}$ & $\mathrm{x}$ \\
\hline acetone & $\mathrm{x}$ & $\mathrm{x}$ \\
\hline acetylene & & \\
\hline acrylic acid (ester grade) & & \\
\hline
\end{tabular}




\begin{tabular}{|l|c|c|}
\hline acrylonitrile & $\mathrm{x}$ & $\mathrm{X}$ \\
\hline adipic acid & & \\
\hline adiponitrile & & \\
\hline alkylate & & \\
\hline alkylbenzene & & \\
\hline ammonia & & \\
\hline ammonium bisulfate & & \\
\hline ammonium sulfate & & \\
\hline aniline & & \\
\hline asphalt & & \\
\hline aviation gasoline & $\mathrm{x}$ & $\mathrm{x}$ \\
\hline benzene & $\mathrm{x}$ & $\mathrm{x}$ \\
\hline bisphenol A & & \\
\hline butadiene & & \\
\hline 1,4-butanediol & & \\
\hline butyl acetate & & \\
\hline butyl rubber & $\mathrm{x}$ & $\mathrm{x}$ \\
\hline C9+ aromatics & & \\
\hline caprolactam & & \\
\hline carbon black HAF & $\mathrm{x}$ & $\mathrm{x}$ \\
\hline caustic soda, 50\% & & \\
\hline chlorine & $\mathrm{x}$ & $\mathrm{x}$ \\
\hline coking & $\mathrm{x}$ & \\
\hline cumene & & \\
\hline cyclohexane & & \\
\hline DDGS dry & & \\
\hline dibasic acids, mixed & & \\
\hline dichloropropylenes & & \\
\hline diethanolamine & & \\
\hline diethylene glycol & & \\
\hline dimethyl terephthalate & & \\
\hline dipropylene glycol & & \\
\hline EPDM rubber & & \\
\hline epichlorohydrin & & \\
\hline epoxy, liquid DGEBPA & \\
\hline ethyl acetate & \\
\hline ethyl acrylate & \\
\hline ethylbenzene & \\
\hline ethylene & \\
\hline ethylene-propylene copolymer & \\
\hline ethylene-VA copolymer & \\
\hline ethylene dichloride & & \\
\hline
\end{tabular}




\begin{tabular}{|l|c|c|}
\hline ethylene glycol & $x$ & $x$ \\
\hline ethylene glycol butyl ethers & & \\
\hline ethylene oxide & $x$ & $x$ \\
\hline 2-ethylhexanol & & \\
\hline 2-ethylhexylacrylate & & \\
\hline fiber & & \\
\hline fuel gas & & \\
\hline gasoline & & \\
\hline germ & & \\
\hline gluten & & \\
\hline hexamethylenediamine & & \\
\hline hydrochloric acid & & \\
\hline isobutane & & \\
\hline isobutanol & & \\
\hline isobutylene & & \\
\hline isobutyraldehyde & $\mathrm{x}$ & $\mathrm{x}$ \\
\hline isopentane & & \\
\hline isopropanol & & \\
\hline kerosene & & \\
\hline kerosene, jet fuel & & \\
\hline linear olefins & & \\
\hline lube & $\mathrm{x}$ & $\mathrm{x}$ \\
\hline m-xylene & $\mathrm{x}$ & $\mathrm{x}$ \\
\hline maleic anhydride & & \\
\hline methanol & & \\
\hline 2-methyl-1,3-propanediol & $\mathrm{x}$ & $\mathrm{x}$ \\
\hline methyl acrylate & & \\
\hline methylene diphenylene isocyanate & & \\
\hline methyl methacrylate & & \\
\hline methyl t-butyl ether & & \\
\hline mixed xylenes & & \\
\hline n-butanol & & \\
\hline n-butyl acrylate & & \\
\hline n-butylene & & \\
\hline n-butyraldehyde & & \\
\hline n-propanol & & \\
\hline naphtha & & \\
\hline naphtha, heavy & & \\
\hline naphtha, light & & \\
\hline naphthalene & & \\
\hline nitrile barrier resin & & \\
\hline nitrobenzene & & \\
\hline
\end{tabular}




\begin{tabular}{|c|c|c|}
\hline o-xylene & $\mathrm{x}$ & $\mathrm{x}$ \\
\hline p-xylene & $\mathrm{x}$ & $\mathrm{x}$ \\
\hline phenol & $x$ & \\
\hline phthalic anhydride & $x$ & $\mathrm{x}$ \\
\hline plasticizer & $x$ & $\mathrm{x}$ \\
\hline \multicolumn{3}{|l|}{ polyamid (nylon 6) } \\
\hline \multicolumn{3}{|l|}{ polyamid (nylon 66) } \\
\hline polybutadiene & $\mathrm{x}$ & $\mathrm{x}$ \\
\hline polycarbonate & $\mathrm{x}$ & \\
\hline \multicolumn{3}{|l|}{ polychloroprene } \\
\hline polyester, unsaturated & $x$ & $\mathrm{x}$ \\
\hline polyester fiber & $\mathrm{x}$ & $\mathrm{x}$ \\
\hline polyester film & $\mathrm{x}$ & $\mathrm{x}$ \\
\hline \multicolumn{3}{|l|}{ polyester polymer } \\
\hline polyethylene, HD & $x$ & $\mathrm{x}$ \\
\hline \multicolumn{3}{|l|}{ polyethylene, HDBM } \\
\hline polyethylene, LD & $\mathrm{x}$ & $\mathrm{x}$ \\
\hline polyethylene, LLD & $\mathrm{x}$ & $\mathrm{x}$ \\
\hline polyethylene terephthalate & $\mathrm{x}$ & $\mathrm{x}$ \\
\hline \multicolumn{3}{|l|}{ polyisoprene } \\
\hline \multicolumn{3}{|l|}{$\begin{array}{l}\text { polymeric methylene diphenylene } \\
\text { isocyanate }\end{array}$} \\
\hline poly(methyl methacrylate) & $x$ & $\mathrm{x}$ \\
\hline \multicolumn{3}{|l|}{ polyol } \\
\hline polypropylene & $\mathrm{x}$ & $\mathrm{x}$ \\
\hline \multicolumn{3}{|l|}{ polypropylene copolymer } \\
\hline polystyrene & $\mathrm{x}$ & $\mathrm{x}$ \\
\hline polystyrene, exp & $x$ & $\mathrm{x}$ \\
\hline \multicolumn{3}{|l|}{ polyvinyl alcohol } \\
\hline polyvinyl chloride & $\mathrm{x}$ & $\mathrm{x}$ \\
\hline propylene & $\mathrm{x}$ & $\mathrm{x}$ \\
\hline \multicolumn{3}{|l|}{ propylene dichloride } \\
\hline \multicolumn{3}{|l|}{ propylene glycol } \\
\hline \multicolumn{3}{|l|}{ propylene glycol ethers } \\
\hline \multicolumn{3}{|l|}{ propylene oxide } \\
\hline \multicolumn{3}{|l|}{ pyrolysis gasoline } \\
\hline \multicolumn{3}{|l|}{ refinery gas } \\
\hline \multicolumn{3}{|l|}{ residual oil, atmospheric } \\
\hline \multicolumn{3}{|l|}{ residual oil, vacuum } \\
\hline SAN resin & $x$ & $\mathrm{x}$ \\
\hline $\begin{array}{l}\text { styrene-butadiene block copolymer, star } \\
\text { block }\end{array}$ & & \\
\hline
\end{tabular}




\begin{tabular}{|l|c|c|}
\hline styrene & $\mathrm{x}$ & $\mathrm{x}$ \\
\hline styrene-butadiene block copolymer & & \\
\hline styrene-butadiene rubber & $\mathrm{x}$ & $\mathrm{x}$ \\
\hline sulfur & & \\
\hline sulfuric acid, dilute & & \\
\hline t-butanol & $\mathrm{x}$ & $\mathrm{x}$ \\
\hline terephthalic acid & & \\
\hline tetrahydrofuran & & \\
\hline toluene & & \\
\hline toluene diamine, crude & $\mathrm{X}$ & $\mathrm{x}$ \\
\hline toluene diisocyanate & & \\
\hline tripropylene glycol & & \\
\hline vinyl acetate & $\mathrm{x}$ & $\mathrm{x}$ \\
\hline vinyl chloride & & \\
\hline water & & \\
\hline wax & & \\
\hline
\end{tabular}

\section{Impact of Omega on Technology Selection}

Figure S1 shows the response of production level to the value of omega. As described in the main text, omega is weighting factor between production and shipment cost, and larger value of omega means shipment cost becomes more dominant. At very low shipment cost (small omega values), refining becomes 99795 KTA, matching the supply constraint. When value of the omega increases, refinery production decreases. A likely explanation is that refining is scattered across the country and refinery units that are farther away from other chemical industrial units become less attractive to operate. While the objective function would encourage gasoline production at these distributed refineries, many chemical feedstocks from refineries would require transportation costs to send those materials to chemical units. The refinery products with the largest mass yields (like residual oil, kerosene, gasoline, naphtha) are not used in chemical processes in this model. The model used materials such as propylene, toluene, xylene, butylene, and ethylene from refining, although mass yields of these are very small.

Another interesting result is that steam cracking continues to operate at higher omega values (higher shipment cost), when other processes reduced their production level. Steam cracking is an upstream process and reducing production level likely has a large cascading effect to subsequent processes that would be forced to shut down without access to steam cracking products. Feed for cracking are primary raw materials and have no shipping cost. It may also be that processes that utilize ethylene and cracking byproducts may be nearby, so shipment costs are low to start with. 
Production Level for Each Process with Varying Value of Omega

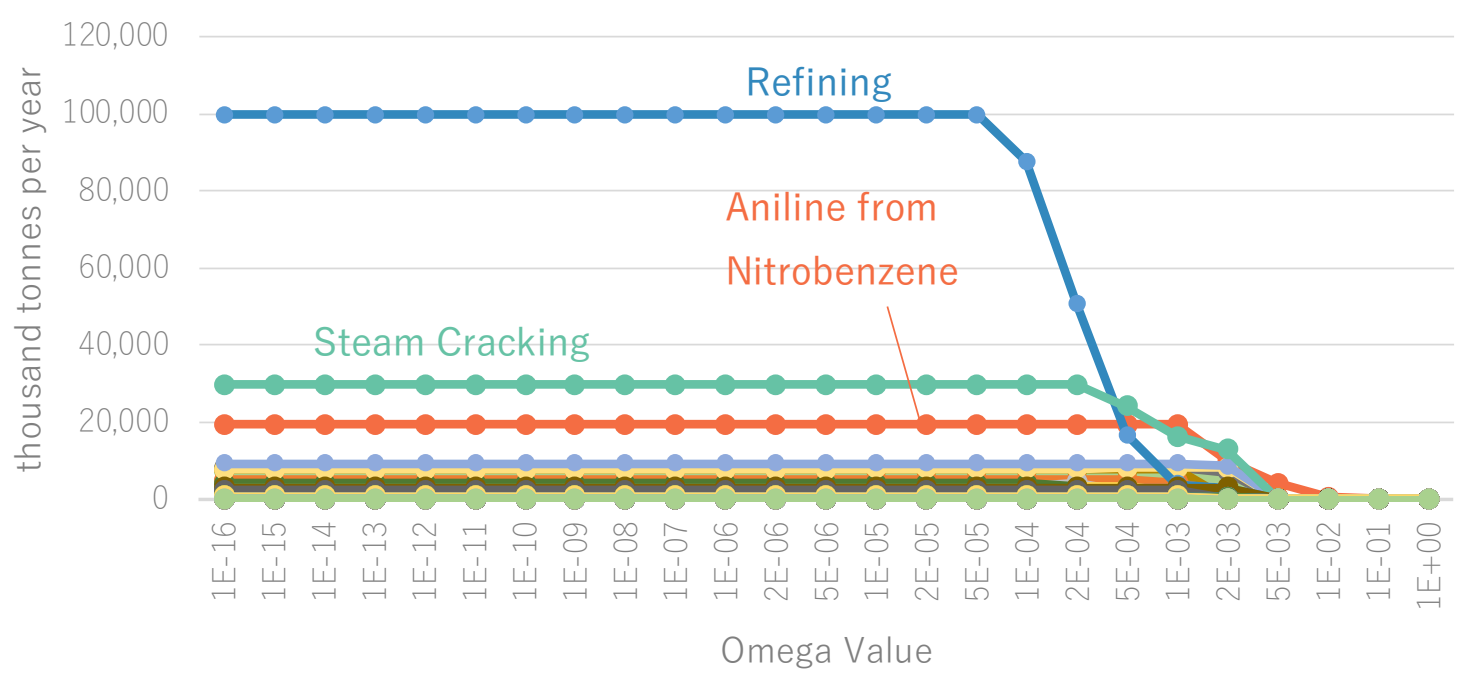

Figure S1. Production level of selected processes for different omega values with the mass production benefit function.

\section{Modeled Effects of Assumed Capacity Disruptions by Geographic Storm Track: Expanded Results}

Detailed results for the map shown in Figure 17 in the main text are provided in the tables below. Table S4 and Table S5 list the magnitude of total chemical production lost and gained by state during secondary disruptions. 
Table S6 shows the primary production chemicals that had a change in production during the disruption.

California shows the largest magnitude of lost production, which is interesting because of its distance from the disruption region. In this model, California has 40 chemical units that primarily produce polymer end products. However, with only one propylene facility (INEOS Carson), the units in California are heavily reliant on raw material supplies from the Gulf Coast.

Table S4. Magnitude of total chemical production lost by state during secondary disruptions in the assumed capacity disruption by geographic storm track scenario.

\begin{tabular}{|c|c|c|}
\hline State & $\begin{array}{l}\text { Lost Production } \\
\text { During Disruption } \\
\text { (thousand tonnes) }\end{array}$ & Primary Products \\
\hline California & 189 & $\begin{array}{l}\text { epoxy liquid DGEBPA, gasoline, polyester unsaturated, } \\
\text { polymethyl methacrylate, polyol, polystyrene, propylene }\end{array}$ \\
\hline Export & 156 & $\begin{array}{l}\text { acetic acid export, acrylonitrile export, cumene export, } \\
\text { cyclohexane export, ethylene-VA copolymer export, } \\
\text { ethylene glycol export, 2-ethylhexanol export, p-xylene } \\
\text { export, phenol export, polybutadiene export, polymethyl } \\
\text { methacrylate export, styrene export, vinyl chloride export }\end{array}$ \\
\hline Louisiana & 76 & $\begin{array}{l}\text { butyl rubber, epoxy liquid DGEBPA, ethylene-VA } \\
\text { copolymer, ethylene oxide, 2-ethylhexylacrylate, linear } \\
\text { olefins, nitrile barrier resin, HDPE, LDPE, LLDPE, } \\
\text { propylene glycol }\end{array}$ \\
\hline South Carolina & 40 & $\begin{array}{l}\text { dimethyl terephthalate, nylon } 66 \text {, polyester polymer, } \\
\text { polyethylene terephthalate, terephthalic acid }\end{array}$ \\
\hline Ohio & 40 & $\begin{array}{l}\text { ABS resin, nitrile barrier resin, phenol, polymethyl } \\
\text { methacrylate, polystyrene, polystyrene-exp, propylene, } \\
\text { SAN resin, styrene-butadiene block copolymer }\end{array}$ \\
\hline Delaware & 39 & ethylene oxide, gasoline \\
\hline Illinois & 27 & ABS resin, LDPE, LLDPE, polystyrene \\
\hline Alabama & 22 & epoxy liquid DGEBPA, phenol, polystyrene \\
\hline Texas & 14 & ethylene-VA copolymer, LDPE, polyvinyl alcohol \\
\hline Pennsylvania & 11 & $\begin{array}{l}\text { phenol, polystyrene, polystyrene-exp, SB block } \\
\text { copolymer-star-block }\end{array}$ \\
\hline Florida & 9 & Adipic acid, epoxy liquid DGEBPA, nylon 66 \\
\hline
\end{tabular}




\begin{tabular}{|l|l|l|}
\hline Mississippi & 9 & $\begin{array}{l}\text { aniline, polymethyl methacrylate, polyvinyl chloride, } \\
\text { SAN resin }\end{array}$ \\
\hline Michigan & 9 & epoxy liquid DGEBPA, polyol, polystyrene, san resin \\
\hline Tennessee & 8 & $\begin{array}{l}\text { dimethyl terephthalate, ethyl acetate, phenol, nylon 66, } \\
\text { polymethyl methacrylate, SB block copolymer-star block }\end{array}$ \\
\hline Kentucky & 8 & $\begin{array}{l}\text { nitrile barrier resin, polybutadiene, polymethyl } \\
\text { methacrylate, polystyrene, propylene glycol, SAN resin, } \\
\text { styrene-butadiene rubber }\end{array}$ \\
\hline Indiana & 6 & phenol \\
\hline Iowa & 5 & LDPE \\
\hline New York & 4 & polystyrene \\
\hline Virginia & 4 & caprolactam, nylon 66, polymethyl methacrylate \\
\hline West Virginia & 4 & nylon 66, polymethyl methacrylate, polyol \\
\hline Connecticut & 3 & polystyrene \\
\hline Wisconsin & 2 & SB block copolymer-star block \\
\hline Georgia & 1 & PMMA \\
\hline North Carolina & 1 & nylon 66, polyol, SB block copolymer-star block \\
\hline Washington & 0.4 & unsaturated polyester \\
\hline New Jersey & 0.2 & epoxy liquid DGEBPA \\
\hline Rhode Island & 0.004 & polyester polymer \\
\hline
\end{tabular}

Table S5. Magnitude of total chemical production gained by state during secondary disruptions in the assumed capacity disruption by geographic storm track scenario.

\begin{tabular}{|l|l|l|}
\hline State & $\begin{array}{l}\text { Gained } \\
\text { Production } \\
\text { During Disruption } \\
\text { (thousand tonnes) }\end{array}$ & Primary Chemicals \\
\hline Louisiana & 14 & $\begin{array}{l}\text { aniline, butadiene, linear olefins, methylene } \\
\text { diphenylene isocyanate, plasticizer, HDPE }\end{array}$ \\
\hline West Virginia & 6 & polyester polymer, polyethylene terephthalate \\
\hline
\end{tabular}




\begin{tabular}{|l|l|l|}
\hline Iowa & 5 & HDPE \\
\hline Export & 3 & $\begin{array}{l}\text { ethylene export, phthalic anhydride export, } \\
\text { terephthalic acid export }\end{array}$ \\
\hline South Carolina & 2 & polyester polymer, polyethylene terephthalate \\
\hline North Carolina & 1 & polyester polymer \\
\hline Tennessee & 1 & plasticizer \\
\hline Illinois & 0.2 & plasticizer \\
\hline
\end{tabular}


Table S6. Basis chemicals that experienced lost production during secondary disruptions in the assumed capacity disruption by geographic storm track scenario.

\begin{tabular}{|l|l|}
\hline Basis Chemical & $\begin{array}{l}\text { Lost } \\
\text { production } \\
\text { During } \\
\text { Disruption } \\
\text { (thousand } \\
\text { tonnes) }\end{array}$ \\
\hline gasoline & 217 \\
\hline polystyrene & 61 \\
\hline p-xylene export & 44 \\
\hline LDPE & 44 \\
\hline phenol & 37 \\
\hline acetic acid export & 27 \\
\hline LLDPE & 22 \\
\hline vinyl chloride export & 21 \\
\hline styrene export & 15 \\
\hline poly(methyl methacrylate) & 15 \\
\hline terephthalic acid & 14 \\
\hline ethylene oxide & 12 \\
\hline ethylene glycol export & 11 \\
\hline dimethyl terephthalate & 11 \\
\hline acrylonitrile export & 9 \\
\hline cyclohexane export & 8 \\
\hline polyamid (nylon 66) & 7 \\
\hline ethylene-VA copolymer & 7 \\
\hline adipic acid & 6 \\
\hline phenol export & 6 \\
\hline styrene-butadiene block copolymer & 6 \\
\hline polyethylene terephthalate & 6 \\
\hline epoxy, liquid DGEBPA & 6 \\
\hline polyvinyl chloride & 6 \\
\hline butyl rubber & 5 \\
\hline polystyrene, exp & 5 \\
\hline cumene export & 5 \\
\hline SB block copolymer, star block & 5 \\
\hline polyol & 4 \\
\hline SAN resin & 4 \\
\hline 2-ethylhexanol export & 4 \\
\hline ABS resin & 3 \\
\hline propylene glycol & 3 \\
\hline polybutadiene export & 2 \\
\hline
\end{tabular}




\begin{tabular}{|l|l|}
\hline ethylene-VA copolymer export & 2 \\
\hline polyester, unsaturated & 2 \\
\hline nitrile barrier resin & 2 \\
\hline propylene & 2 \\
\hline polyester polymer & 2 \\
\hline styrene-butadiene rubber & 2 \\
\hline linear olefins & 1 \\
\hline ethylacetate & 1 \\
\hline polyvinyl alcohol & 1 \\
\hline 2-ethylhexyl acrylate & 1 \\
\hline polybutadiene & 0.3 \\
\hline caprolactam & 0.3 \\
\hline polymethyl methacrylate export & 0.2 \\
\hline aniline & -0.0004 \\
\hline ethylene export & -0.01 \\
\hline butadiene & -0.1 \\
\hline methylene diphenylene isocyanate & -0.3 \\
\hline phthalic anhydride export & -0.4 \\
\hline terephthalic acid export & -2 \\
\hline plasticizer & -3 \\
\hline HDPE & -7 \\
\hline & \\
\hline
\end{tabular}

